\title{
Perspectives of lung cancer control and molecular prevention
}

\author{
Darrell D Davidson ${ }^{1} \&$ Liang Cheng*,1 (iD) \\ ${ }^{1}$ Department of Pathology \& Laboratory Medicine, Indiana University School of Medicine, Indianapolis, Indiana, IN 46202, USA \\ *Author for correspondence: Tel.: +1 317491 6442; Fax +1 317491 6419; liang_cheng@yahoo.com
}
"What would be needed for such a screening program to succeed and be widely accepted? First, there should be a global detection system to identify patients with early lesions before invasion has occurred"”

First draft submitted: 28 August 2019; Accepted for publication: 6 September 2019; Published online: 25 October 2019

Keywords: biomarker $\bullet$ carcinogenesis $\bullet$ lung • molecular prevention $\bullet$ precision genomics $\bullet$ screening and cancer control

\section{Highlights}

Lung cancer is comprised of multiple histologic types, the three most common being squamous cell carcinoma, adenocarcinoma and small cell carcinoma. These derive from different cell types and generally occupy different locations within the broncopulmonary tree. Many lung cancers are multifocal, apparently arising from a premalignant clonal 'field affect' change distributed throughout the lung [1-3]. Although the latent phase appears to be similar to that of cervical carcinoma, the premalignant cells are difficult to identify by morphology alone, so precision genomic tools are essential for studying the earliest stages of lung cancer evolution [4]. Furthermore, lung cancer tends to metastasize at an early stage, and to spread by both lymphatic and hematogenous routes. Predicting which preinvasive clones are likely to develop this destructive potential is another prospect of precision genomic studies [5]. Although over $60 \%$ of breast and prostate carcinomas appear at a curable early stage, $57 \%$ of lung carcinoma cases present with distant metastases, causing 5-year survival to be $19 \%$ or less [6]. Precision genomics is illuminating early changes that permit cancer evolution, invasion, immunosuppression and metastasis $[5,7]$.

The three frequent types of lung cancer are so different in location, oncogenic mutation sequences and precursor lesions that lung cancer screening programs must comprise two or three screening strategies combined [8]. Incipient squamous cell carcinoma and possibly small cell carcinoma may be detected with the bronchoscope, but adenocarcinoma precursors in the lung periphery are only detected by computed tomography. The 53,454-participant National Lung Screening Trial in 2013 reported a 20\% reduction of mortality for the cohort screened by low dose computed tomography (LDCT) three-times a year [9]. However, more mature data and expansion of LDCT screening to the general healthcare setting now show only $16 \%$ reduction in mortality [10]. There has also been only $3.9 \%$ acceptance of screening by the mature heavy smoker population, despite provision in the Affordable Care Act (ACA) mandating insurance coverage for this preventive service to at-risk patients [11]. Moreover, it appears that complication rates from biopsy procedures relating to LDCT in general use are about twice as high as those seen during the National Lung Screening Trial protocol [10]. Yet the sensitivity continues to improve and the cost continues to decline for LDCT image processing as artificial intelligence tools to streamline LDCT for population screening advance [12]. Future developments should not only optimize the frequency and duration of screening, and preselection of patients to screen, but also answer questions about whom to biopsy when abnormalities are found, as well as the safest biopsy procedures, and best use of biopsy results based on a precision genomics [13,14].

Preinvasive carcinoma in proximal airways is seldom discovered by radiographic techniques. Autofluorescence bronchoscopy screening programs can usually identify preinvasive squamous lesions. LDCT, on the other hand, detects adenocarcinoma in situ, and other premalignant changes in peripheral glandular cells. Effective tools to discover early small cell carcinoma are also needed, but the screening interval would have to be short, on the order of months, to descry this rapidly progressing, lethal type of lung cancer. And a challenge for bronchoscopic squamous 
abnormality screening is that preinvasive squamous cell carcinoma (CIS) progresses to invasive carcinoma only $50 \%$ of the times $[5,15]$. Although CIS lesions are easily ablated once identified, it would reduce morbidity to treat only the 50\% destined to become cancer [4]. Similar progression prediction and grading problems also occur with preinvasive glandular lesions found by LDCT in the lung periphery, such as atypical adenomatous hyperplasia. Identifying the need-to-treat group will probably require a tissue biopsy for precision genomic and epigenetic testing for predictors of invasion [5].

Hence, what is needed is an inexpensive, noninvasive, easily marketed, sensitive test to determine which patients should undergo LDCT or bronchoscopy. These expensive confirmatory tests would then be used to identify patients with lesions that should be biopsied, rather than for initial screening. Attempts to find serum or molecular markers with high sensitivity for preinvasive cancer have been disappointing, and open to criticism, because most studies use early stage invasive carcinoma rather than carcinoma in situ or dysplasia tissue for biomarker discovery $[4,16]$. Preinvasive carcinoma, by definition, is separated by the basement membrane from blood vessels and lymphatics. It is, therefore, not surprising that studies of serum markers for lung cancer fail to detect preinvasive stages. To achieve convenient global sampling of in situ bronchial and alveolar epithelial changes in a manner analogous to the Papanicolau test, bronchial washings could be performed without deep sedation or anesthesia [17]. Such nonbronchoscopic specimens have shown good correlation with bronchoscopic lavage samples for identifying ventilator-associated pneumonia [18]. These washings, however, may sample only portions of the complex tracheobronchial system, whereas genomic changes in the epithelia may be localized in a different region of the labyrinth [19]. The procedure itself, although less complex than bronchoscopy, still may be intrusive enough to have poor acceptance by patients. If such global pulmonary premalignant fluid markers exist, precision genomic analysis, probably supported by artificial intelligence assessment of the molecular data, will be needed to detect them with high sensitivity and specificity in all patients.

Although lung cancer stubbornly refuses to begin at a single predictable site like cervical cancer, there is yet an advantage that the entire tracheobronchial tree communicates with a single airway and there is continuous gas exchange from every part of the lung [20]. One of the hallmarks of cancer from the very earliest stages is metabolic alteration [7]. It would be reasonable to anticipate distinctive volatile metabolite production from different types of transformed cells. These metabolites could be detected in exhaled gases from a patient with preinvasive lung cancer. In multiple studies, dogs have shown the ability to detect malignancy by sniffing exhaled air, serum and urine from patients with a variety of cancers [21]. Only one recent study focused primarily on non-small-cell lung cancer, for which the three well-trained beagle dogs in the study using a 4:1 control-to-positive design, had overall sensitivity of 96.7\% for detecting serum from patients with invasive lung cancer [22]. It may be possible to condense nongaseous components of exhaled air from patients in a lung cancer screening trial to increase the sensitivity of trained dogs to detect in situ carcinoma. The condensed material could also be fractionated to identify which volatile components the dog is able to detect and associate with malignancy. Instruments would soon follow that could detect the appropriate analytes at 'dog sniffer' concentrations [23]. The sequence for screening selected populations would then be: breathalyzer, bronchoscopy and LDCT, biopsy, molecular analysis and precision treatment. Information gained from studying early lung carcinoma may then eventually lead to public health measures based on the underlying molecular causes of chemical-induced, tobacco-related cancers [24,25].

What would be needed for such a screening program to succeed and be widely accepted? First, there should be a global detection system to identify patients with early lesions before invasion has occurred. This should be inexpensive, safe and attractive to both patients and their primary care physicians [26]. Second, there should be improved techniques for identifying the proper biopsy site for patients identified by the global detection system. The best biopsy location site techniques would not only identify where to recover tissue, but also yield corroborative attestation from the gross appearance of the lesion $[12,27]$. The gross features would play a role similar to that provided by colposcopy in the cervix. Third, evidence to predict the lesion's future behavior should be based both on radiographic analysis using artificial intelligence, and on precision genomics of biopsy materials [4]. Artificial intelligence will aid not only for identifying the site to biopsy, but also for grading and predicting how likely a lesion is to progress [12,28]. Finally, once progression inclined lesions are identified; treatments to remove the abnormal cells with enhanced safety and minimization of tissue damage will be needed. Systemic therapies are already showing promise in this area [17,29]. At this point a wealth of tissue from preinvasive lung epithelia will allow understanding of the earliest triggers of lung carcinogenesis. With this knowledge it will be possible to prevent lung carcinoma on a public health scale. 


\section{Financial \& competing interests disclosure}

The authors have no relevant affiliations or financial involvement with any organization or entity with a financial interest in or financial conflict with the subject matter or materials discussed in the manuscript. This includes employment, consultancies, honoraria, stock ownership or options, expert testimony, grants or patents received or pending, or royalties.

No writing assistance was utilized in the production of this manuscript.

\section{References}

1. Steiling K, Ryan J, Brody JS, Spira A. The field of tissue injury in the lung and airway. Cancer Prev. Res. (Phila.) 1(6), 396-403 (2008).

2. Cheng L, Alexander RE, Maclennan GT et al. Molecular pathology of lung cancer: key to personalized medicine. Mod. Pathol. 25(3), 347-369 (2012).

3. Wang X, Wang M, Maclennan GT et al. Evidence for common clonal origin of multifocal lung cancers. J. Natl Cancer Inst. 101(8), 560-570 (2009).

4. Campbell JD, Mazzilli SA, Reid ME et al. The case for a Pre-Cancer Genome Atlas (PCGA). Cancer Prev. Res. (Phila.) 9(2), 119-124 (2016).

5. Teixeira VH, Pipinikas CP, Pennycuick A et al. Deciphering the genomic, epigenomic, and transcriptomic landscapes of pre-invasive lung cancer lesions. Nat. Med. 25(3), 517-525 (2019).

6. Siegel RL, Miller KD, Jemal A. Cancer statistics, 2019. CA Cancer J. Clin. 69(1), 7-34 (2019).

7. Hanahan D, Weinberg RA. Hallmarks of cancer: the next generation. Cell 144(5), 646-674 (2011).

8. Loomans-Kropp HA, Umar A. Cancer prevention and screening: the next step in the era of precision medicine. NPJ Precision Oncol. 3, 3 (2019).

9. Aberle DR, Adams AM, Berg CD et al. Reduced lung-cancer mortality with low-dose computed tomographic screening. N. Engl. J. Med. 365(5), 395-409 (2011).

10. Huo J, Shih YT. Complication rates in a study of invasive diagnostic procedures for lung abnormalities-in reply. JAMA Intern. Med. 179(6), 847 (2019).

11. Lei F, Lee E. Barriers to lung cancer screening with low-dose computed tomography. Oncol. Nurs. Forum 46(2), E60-E71 (2019).

12. Ardila D, Kiraly AP, Bharadwaj $S$ et al. End-to-end lung cancer screening with three-dimensional deep learning on low-dose chest computed tomography. Nat. Med. 25(6), 954-961 (2019).

13. Field JK, Oudkerk M, Pedersen JH, Duffy SW. Prospects for population screening and diagnosis of lung cancer. Lancet 382(9893), 732-741 (2013).

14. Tammemagi MC, Ten Haaf K, Toumazis I et al. Development and validation of a multivariable lung cancer risk prediction model that includes low-dose computed tomography screening results: a secondary analysis of data from the national lung screening trial. JAMA Network Open 2(3), e190204 (2019).

15. Izumchenko E, Chang X, Brait $\mathrm{M}$ et al. Targeted sequencing reveals clonal genetic changes in the progression of early lung neoplasms and paired circulating DNA. Nat. Commun. 6, 8258 (2015).

16. Jamal-Hanjani M, Wilson GA, Mcgranahan N et al. Tracking the evolution of non-small-cell lung cancer. N. Engl. J. Med. 376(22), 2109-2121 (2017).

17. Spira A, Yurgelun MB, Alexandrov L et al. Precancer atlas to drive precision prevention trials. Cancer Res. 77(7), 1510-1541 (2017).

18. Afify MH, Shaheen EA, El-Dahdouh SS, El-Feky HMJEJOCD, Tuberculosis. Comparison between bronchoscopic BAL and non-bronchoscopic BAL in patients with VAP. 65(1), 113-119 (2016).

19. Tuo L, Sha S, Huayu Z, Du K. P16(INK4a) gene promoter methylation as a biomarker for the diagnosis of non-small cell lung cancer: an updated meta-analysis. Thoracic Cancer 9(8), 1032-1040 (2018).

20. Arendt D, Musser JM, Baker CVH et al. The origin and evolution of cell types. Nat. Rev. Genet. 17(12), 744-757 (2016).

21. Pirrone F, Albertini MJJOVB. Olfactory detection of cancer by trained sniffer dogs: a systematic review of the literature. $J$ Vet Bahav. 19, 105-117 (2017).

22. Junqueira H, Quinn TA, Biringer R et al. Accuracy of canine scent detection of non-small cell lung cancer in blood serum. J. Am. Osteopath. Assoc. (2019). doi:10.7556/jaoa.2019.077 (Epub ahead of print).

23. Brooks SW, Moore DR, Marzouk EB, Glenn FR, Hallock RM. Canine olfaction and electronic nose detection of volatile organic compounds in the detection of cancer: a review. Cancer Invest. 33(9), 411-419 (2015).

24. Kensler TW, Spira A, Garber JE et al. Transforming cancer prevention through precision medicine and immune-oncology. Cancer Prev. Res. (Phila.) 9(1), 2-10 (2016).

25. Maresso KC, Tsai KY, Brown PH, Szabo E, Lippman S, Hawk ET. Molecular cancer prevention: current status and future directions. $C A$ Cancer J. Clin. 65(5), 345-383 (2015). 
26. Beane J, Mazzilli SA, Tassinari AM et al. Detecting the presence and progression of premalignant lung lesions via airway gene expression. Clin. Cancer Res. 23(17), 5091-5100 (2017).

27. Lambin P, Leijenaar RTH, Deist TM et al. Radiomics: the bridge between medical imaging and personalized medicine. Nat. Rev. Clin. Oncol. 14(12), 749-762 (2017).

28. Patil PD, Hobbs B, Pennell NA. The promise and challenges of deep learning models for automated histopathologic classification and mutation prediction in lung cancer. J. Thorac. Dis. 11(2), 369-372 (2019).

29. Hsu PP, Shaw AT. Lung cancer: a wily genetic opponent. Cell 169(5), 777-779 (2017). 